\title{
Fast-Transient Repetitive Control Strategy for a Three-phase LCL Filter-based Shunt Active Power Filter
}

\author{
Zheng Zeng ${ }^{*}$, Jia-Qiang Yang ${ }^{\dagger}$, Shi-Lan Chen ${ }^{*}$, and Jin Huang ${ }^{*}$ \\ ${ }^{*}$ College of Electrical Engineering, Zhejiang University, Hangzhou, China
}

\begin{abstract}
A fast-transient repetitive control strategy for a three-phase shunt active power filter is presented in this study to improve dynamic performance without sacrificing steady-state accuracy. The proposed approach requires one-sixth of the fundamental period required by conventional repetitive control methods as the repetitive control time delay in the synchronous reference frames. Therefore, the proposed method allows the system to achieve a fast dynamic response, and the program occupies minimal storage space. A proportional-integral regulator is also added to the current control loop to eliminate arbitrary-order harmonics and ensure system stability under severe harmonic distortion conditions. The design process of the corrector in the fast-transient repetitive controller is also presented in detail. The LCL filter resonance problem is avoided by the appropriately designed corrector, which increases the margin of system stability and maintains the original compensation current tracking accuracy. Finally, experimental results are presented to verify the feasibility of the proposed strategy.
\end{abstract}

Key words: Active power filter, Harmonic compensation, Internal model principle, LCL filter, Repetitive control

\section{INTRODUCTION}

With the rapid development of power electronics, a variety of power electronic devices and nonlinear loads have been widely used. Numerous problems associated with reactive power and harmonic pollution have emerged in power systems. Harmonic suppression and reactive power compensation have become popular issues in current research. Traditional passive power filters are gradually being replaced by active power filters (APFs) [1]. A novel APF with an LCL filter [2]-[4] can minimize the amount of distortion current in the utility grid and achieve a fast dynamic response with small inductors and filter capacitors.

A key point in the proper implementation of APF is compensation current tracking. Various methods have been proposed in literature to improve APF current tracking performance [5]-[9]. Hysteresis control [5] and proportional-integral (PI) control [6] are conventional methods commonly employed in industrial APF applications although

Manuscript received Mar. 28, 2013; accepted Dec. 30, 2013

Recommended for publication by Associate Editor Sung-Yeul Park.

†Corresponding Author: yjq1998@163.com

Tel: +86-571-8795-1784, Fax: +86-571-8795-1625, Zhejiang University

*College of Electrical Engineering, Zhejiang University, China new control methods have also been proposed, such as fuzzy control [7], sliding mode variable structure control [8], and adaptive control [9]. Hysteresis control systems are robust although their switch frequency is uncertain; such uncertainty produces harmonics with a continuous spectrum and increases the difficulty of designing an appropriate output filter. PI control has a fast dynamic response and good tracking capability for DC signals. Increasing the PI controller gain helps improve AC signal tracking performance; however, a large controller gain often causes a resonance problem in the LCL filter [10], [11]. The proportion resonance (PR) control method can eliminate the tracking error of regulation sinusoidal signals [12]. However, to compensate for harmonics in this method, each harmonic frequency must correspond to its specific regulator. Therefore, designing a multi-resonant controller for all harmonic components requires a large amount of resources and is thus unsuitable for practical systems.

Repetitive control from the internal model principle provides a high-performance practical solution [13]-[15]. Repetitive control allows for exact asymptotic output tracking of periodic inputs or rejection of periodic disturbances and is thus widely used in inverter waveform control. This approach can meet the necessary high-gain requirements of current control loops in APF [16]-[19]. However, because the control action is 
postponed for one fundamental period, conventional repetitive control often fails to eliminate harmonics with satisfactory dynamic response [20].

Normally, the conventional repetitive controller provides high gains at all harmonic frequencies. Given that most power electronic systems mainly contain odd harmonics, repetitive control schemes intended to compensate for only odd harmonics have been proposed in [21] and [22]. In these odd-harmonic repetitive controllers, time delay is reduced to one half of the fundamental period, which can be regarded as an improvement of system dynamic response. In three-phase power applications, the harmonic components usually concentrate in multiples of $6 n \pm 1$ of the fundamental frequency. The authors of [23] presented a six-model repetitive controller that employs a parallel combination of $(6 n+i)(i=0$, $1,2,3,4,5)$ order harmonic internal models with six independent control gains to eliminate $(6 n+i)$ th harmonics. However, this method increases the complexity of the control loop and consumes a large amount of computing resources; as a result, the method is difficult to apply to actual systems. In [24], the authors presented a repetitive controller in a stationary frame that selects $(6 n \pm 1)$ th $(n=0,1,2, \ldots)$ harmonics for compensation. The $(6 n \pm 1)$ th harmonic repetitive controller obtains enhanced frequency selectivity and has low computation complexity. However, this method cannot effectively compensate for even harmonics because the controller gains are not sufficiently high at these harmonic frequencies.

A fast-transient repetitive controller for a three-phase, LCL filter-based shunt APF is proposed in this study. The controller is implemented in a positive-rotating synchronous frame where all the $(6 n \pm 1)$ th $(n=0,1,2 \ldots)$ harmonics can be well eliminated. The maximum repetitive control delay time is reduced to one-sixth of the fundamental period; therefore, the dynamic response is significantly improved without sacrificing steady-state performance. The PI regulator is also combined with a fast-transient repetitive controller to compensate for even harmonics under unbalanced conditions. Theoretical analysis and deduction of the proposed method are also demonstrated. The design process of the corrector in the proposed fast-transient repetitive controller is presented in detail. The LCL filter resonance problem is avoided by using an appropriately designed corrector that increases the margin of system stability and maintains the original compensation current tracking accuracy. The feasibility of the hybrid current controller is validated by the experimental results.

\section{APF CONTROL SYSTEM AND MATHEMATICAL MODEL}

\section{A. APF Structure and Control System}

The structure of the proposed LCL filter-based shunt APF is shown in Fig. 1. The shunt APF is composed of two-level

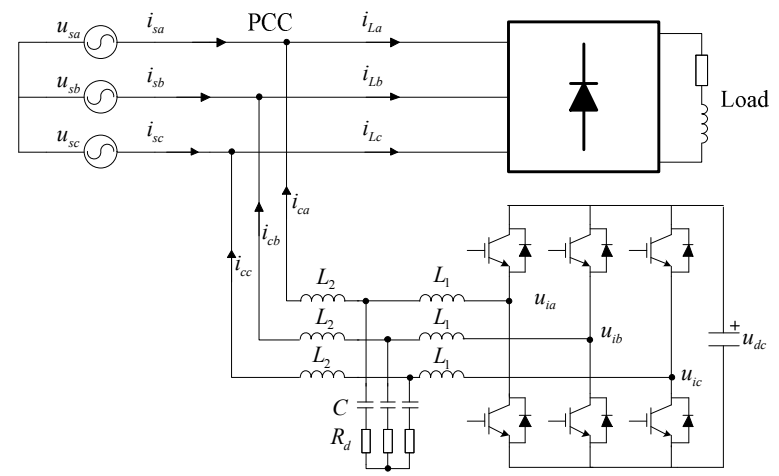

Fig. 1. Structure of shunt APF with LCL filter.

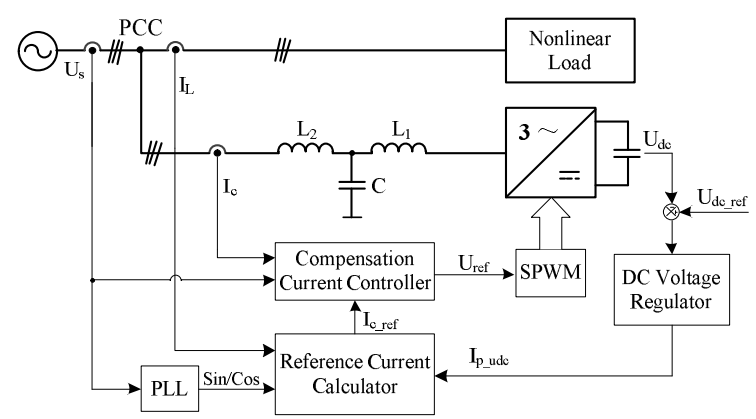

Fig. 2. Block diagram of shunt APF control system.

voltage source inverters with a DC capacitor and LCL-type output filter. $L_{1}$ denotes the inverter-side filter inductors, $L_{2}$ denotes the grid-side filter inductors, $C$ denotes the filter capacitors, and $R_{d}$ denotes the damping resistors. A three-phase diode rectifier with resistive and inductive load is adopted as a harmonic source to produce the harmonic currents.

A block diagram of the shunt APF control system is presented in Fig. 2. The control system contains a reference current calculator, a DC voltage regulator, and a compensation current controller. The reference current calculator detects the harmonic components in the load currents. A DC voltage regulator is adopted to maintain DC bus voltage stability. The compensation reference currents are obtained by adding the output current reference of the DC voltage regulator and the harmonic components in the load current. The compensation current controller generates a reference voltage to allow the compensation current to track the reference current accurately. Therefore, the compensation current can offset the harmonic components in the load currents, and the harmonics in the point of common coupling (PCC) current are also eliminated.

Considering that the inductor resistance and grid inductance are negligible, the transfer function related to input voltage $u_{i}$ and output current $i_{c}$ can be deduced as

$$
G_{L C L}(s)=\frac{i_{c}}{u_{i}}=\frac{R_{d} C s+1}{L_{1} L_{2} C s^{3}+\left(L_{1}+L_{2}\right) R_{d} C s^{2}+\left(L_{1}+L_{2}\right) s}
$$




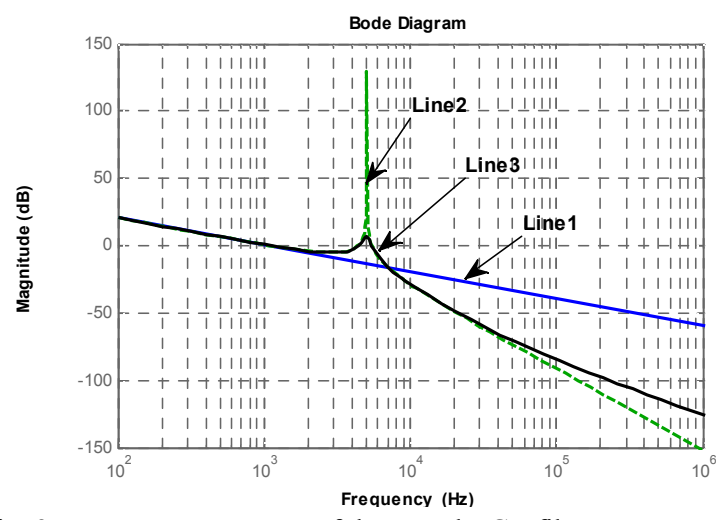

Fig. 3. Frequency response of the L and LCL filters.

Based on transfer function $G_{L C L}(s)$, the LCL filter can be regarded as a three-order system. An LCL filter is more difficult to control than an L filter.

Fig. 3 shows the voltage-current frequency responses of a first-order L filter (Line 1) and a third-order LCL filter (Line 2). Line 3 shows the frequency responses of an LCL filter with a passive damping resistor. A small damping resistor is usually added to the filter capacitor branch to prevent problems in system resonance. The third-order LCL filter should provide an appropriate resonance frequency below the PWM switching frequency, an acceptable level of current ripples in inverter-side inductor $L_{1}$, and low idle reactive power for the apparatus. The detailed LCL filter exemplary design procedure is provided in [2]. In short, the LCL filter has a much better stop-band attenuation than the L filter. Therefore, the output filter of the LCL filter has much lower equivalent inductance than that of the $\mathrm{L}$ filter.

\section{B. Mathematical Model}

Fig. 3 shows that the L and LCL filters have similar magnitude-frequency characteristics within the passband of the filters. For this reason, the capacitor branch of the LCL filter can be disregarded. The LCL filter behaves as an L filter with an inductance value of $L=L_{1}+L_{2}$. The equations of the output current based on Kirchhoff's voltage law result in

$$
\left\{\begin{array}{l}
\left(L_{1}+L_{2}\right) \frac{d i_{c a}}{d t}=u_{i a}-u_{s a}-i_{c a} R \\
\left(L_{1}+L_{2}\right) \frac{d i_{c b}}{d t}=u_{i b}-u_{s b}-i_{c b} R \\
\left(L_{1}+L_{2}\right) \frac{d i_{c c}}{d t}=u_{i c}-u_{s c}-i_{c c} R
\end{array}\right.
$$

where $u_{i a}, u_{i b}$, and $u_{i c}$ are the inverter voltages; $u_{s a}, u_{s b}$, and $u_{s c}$ are the grid voltages; $i_{c a}, i_{c b}$, and $i_{c c}$ are the compensating currents; and $R$ is the equivalent resistance of the inductor.

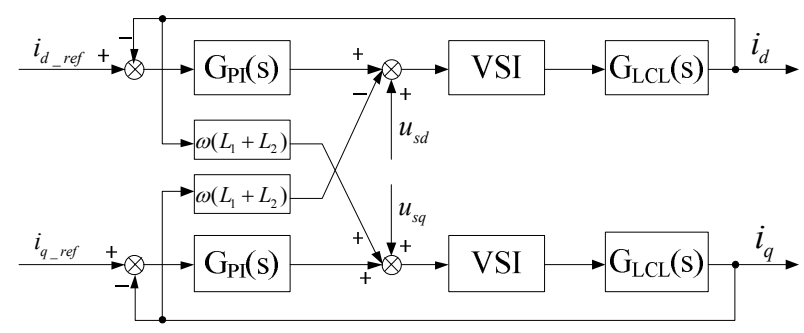

Fig. 4. Current control block diagram of APF in the $d-q$ frame.

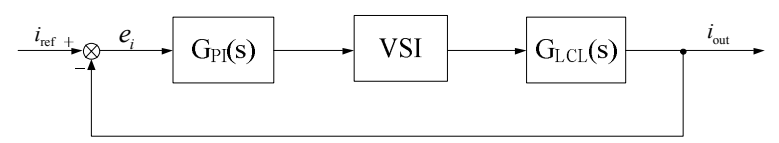

Fig. 5. Simplified current control block diagram.

Using Park transformation, Equation (3) can be obtained by converting the stationary reference frame systematic formulas [Equation (2)] into d-q synchronous reference frame formulas as

$$
\left\{\begin{array}{l}
\left(L_{1}+L_{2}\right) \frac{d i_{c d}}{d t}=u_{i d}-u_{s d}-i_{c d} R+\omega\left(L_{1}+L_{2}\right) i_{c q} \\
\left(L_{1}+L_{2}\right) \frac{d i_{c q}}{d t}=u_{i q}-u_{s q}-i_{c q} R-\omega\left(L_{1}+L_{2}\right) i_{c d}
\end{array} .\right.
$$

Obviously, cross-coupling items exist between the d-axis and q-axis currents.

\section{Decoupling Method with PI Controller}

A controller with feedback cross-decoupling can eliminate the coupling between the $\mathrm{d}$ and $\mathrm{q}$ axes. A grid voltage feed-forward path is added to the controller to eliminate the influence of grid voltage fluctuation. A PI controller with grid voltage feed-forward and output current feedback cross decoupling is shown in Fig. 4.

The decoupling items and the feed-forward voltage can be regarded as disturbances in the above mentioned approaches. The current control block diagram of APF can be simplified as shown in Fig. 5.

The controller requires a wide bandwidth to obtain satisfactory compensation current tracking performance. Owing to the limited sampling frequency and calculation delay time in digital systems, the control variable output is usually retarded by one control period. This condition results in further phase delay. The system phase delay can be reduced by increasing the gain of the PI controller and the sampling and switching frequencies. However, all these methods cannot improve harmonic compensation. Furthermore, the phase delay may cause instability in the LCL filter-based shunt APF. Therefore, a current controller with high stability and high-precision tracking performance is required. 


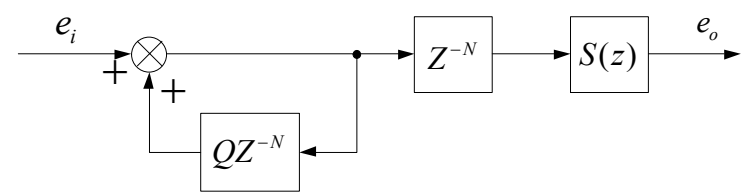

Fig. 6. Conventional repetitive controller structure.

\section{FAST-TRANSIENT REPETITIVE STRATEGY}

\section{A. Review of Conventional Repetitive Controller Structure}

The basic idea behind repetitive control is based on the internal-model principle of control theory. The general structure of a conventional repetitive controller is shown below.

The core of the repetitive controller is a modified internal model denoted by the positive feedback loop in Fig. 6. The original internal model incorporates the unity gain in the positive feedback path so that the tracking error is converged based on its repetition period. However, in practice, the internal model is often modified by incorporating an attenuation coefficient $(Q)$ instead of the unity gain. Although this manipulation results in reduced reference tracking accuracy, it also increases the margin of system stability.

From Fig. 6, the transfer function of the conventional repetitive controller is

$$
G_{R E P}(z)=G_{i m}(z) S(z)=\frac{z^{-N}}{1-Q z^{-N}} S(z)
$$

where $S(z)$ is a corrector designed to compensate for the controlled object according to its characteristic. The period delay part $Z^{-N}$ in the forward channel causes a delay of one fundamental period. Thus, the control effect has a certain foresight of the next period. Owing to a long time delay, the conventional repetitive controller has a very slow transient response speed. For this reason, harmonic current compensation is difficult under conditions of rapid load fluctuations .

\section{B. Proposed Fast-transient Repetitive Controller Structure}

Generally speaking, a balanced three-phase system only contains $(6 n \pm 1)$ th $(n=0,1,2 \ldots)$ harmonics because many power conversion processes (i.e., diode rectifier loads) involved in industrial applications produce harmonic components at these frequencies. In the synchronous frame with $+\omega$ rotation speed, the positive sequences of the $7 \omega, 13 \omega$, $19 \omega \ldots$ signals are transformed to $+6 \omega,+12 \omega,+18 \omega \ldots$ signals, whereas the negative sequences of the $5 \omega, 11 \omega, 17 \omega \ldots$ signals are transformed to $-6 \omega,-12 \omega,-18 \omega \ldots$ signals. The $(6 n \pm 1) \omega$ $(n=0,1,2 \ldots)$ harmonic components transform into $6 n \omega(n=0$, $1,2,3 \ldots)$ signals in the positive synchronous frame. This useful feature simplifies the synchronous frame controller

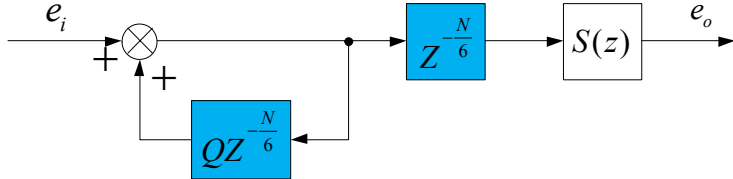

Fig. 7. Fast-transient repetitive controller structure.

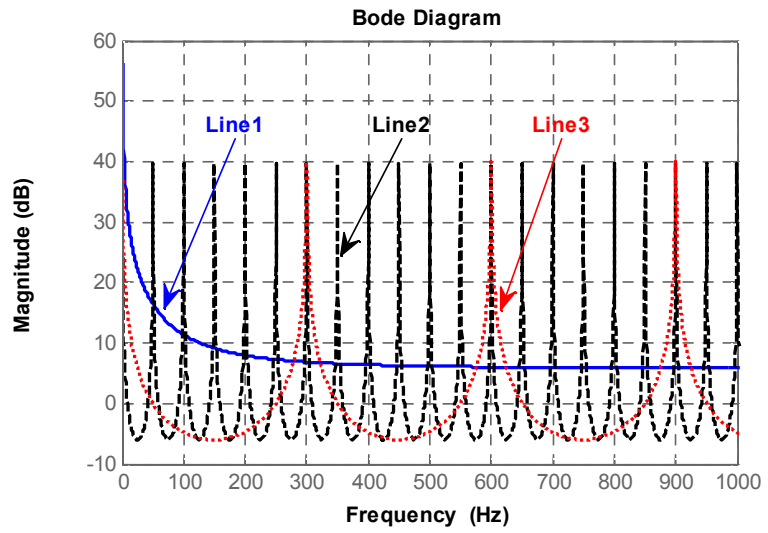

Fig. 8. Frequency response of PI and repetitive control.

design because only the $6 n$th $(n=0,1,2 \ldots)$ harmonics are compensated. Thus, a fast-transient repetitive control strategy is proposed in this study. The strategy aims to compensate for $6 n$th harmonics in a three-phase shunt APF.

The structure of the proposed fast-transient repetitive controller is shown in Fig. 7.

The transfer function of the fast-transient repetitive controller is

$$
G_{R E P}(z)=\frac{z^{-N / 6}}{1-Q z^{-N / 6}} S(z) .
$$

Conventional repetitive controllers are designed to eliminate all odd and even harmonics at the cost of introducing a fundamental period delay. However, from the previous discussions, the time delay required for a fast-transient repetitive controller can be significantly reduced from one fundamental $T$ period to $T / 6$. For this reason, a fast-transient repetitive controller provides faster dynamic performance than the conventional one. In addition, by considering only ( $6 n \pm$ 1)th harmonics, the proposed repetitive controller can avoid the amplification of other harmonics to the PCC currents.

Fig. 8 shows the open-loop frequency responses of the PI controller (Line 1), conventional repetitive controller (Line 2), and proposed fast-transient repetitive controller (Line 3). The PI controller is implemented with parameters $k_{p}=2$ and $k_{i}=$ 2000. The gain of the PI controller for the DC signal is above $50 \mathrm{~dB}$, but its gain in the harmonic-frequency range cannot satisfy the harmonic compensation requirements. The conventional repetitive controller is implemented with a delay of one fundamental period, where $N=300$ and $Q=0.99$. Fig. 8 shows that the conventional repetitive controller provides high 


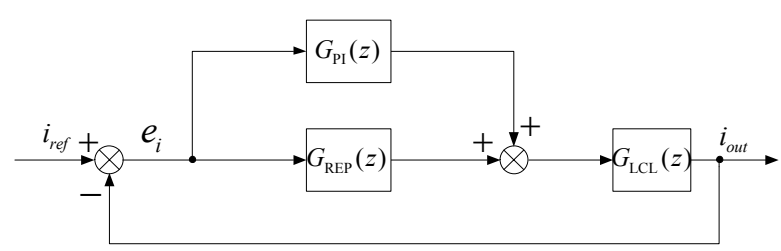

Fig. 9. Hybrid controller scheme for APF with an LCL filter.

gain $(40 \mathrm{~dB})$ at each harmonic frequency, whereas the corresponding resonant frequencies of the fast-transient repetitive controller $(N=300, Q=0.99)$ are only in the 6 th harmonics.

Given that the repetitive control strategy contains a necessary delay section, satisfying the requirement of fast dynamic response and stability of APF is difficult. Thus, a hybrid current controller that consists of PI and repetitive controllers is implemented in the current loop. The PI controller improves the dynamic performance and maintains system stability, whereas the repetitive controller provides high gain in harmonics to ensure that the PCC current remains sinusoidal.

To achieve a hybrid output control, the PI and repetitive controllers are connected in parallel in the forward path of the current control loop as shown in Fig. 9.

The PI controller exports a thin control quantity in the steady-state condition because of a few tracking errors. As a result, the hybrid control parameter is principally determined by the repetitive controller. However, when the system undergoes a severe dynamic load fluctuation, the PI controller plays the major role in the hybrid control system and the repetitive controller output quantity cannot adjust promptly. After the delay, the repetitive controller gradually decreases the tracking error and thus weakens the influence of the PI controller. Eventually, the system achieves a new steady state.

When the grid voltage or load condition is unbalanced, each $(6 \mathrm{n} \pm 1)$ th harmonic component contains both positive and negative sequences. Therefore, the 6 nth harmonics as well as other orders of harmonics are visible in the positive synchronous frame. However, in most cases, the unbalanced condition of the grid voltage or load is not severe (mostly below $10 \%$ ). In such cases, the PI controller inside the hybrid control strategy provides additional gain for the negative sequence harmonics.

\section{Compensation Current Tracking Accuracy Analysis}

The repetitive control method introduced to the hybrid current controller improves the tracking accuracy and eliminates the steady state error. By analyzing the error transfer function, the tracking accuracy of the repetitive control method can be compared with that of other methods. As shown in Fig. 5, the error transfer function with a PI controller is as follows:

$$
\frac{e_{i}(z)}{i_{r e f}(z)}=\frac{1}{1+G_{P I}(z) G_{L C L}(z)} .
$$

The error transfer function with a hybrid controller is as follows:

$$
\left\{\begin{array}{c}
\frac{e_{i}(z)}{i_{r e f}(z)}=\frac{1}{1+\left[G_{R E P}(z)+G_{P I}(z)\right] G_{L C L}(z)} \\
G_{R E P}(z)=\frac{z^{-N / 6}}{1-Q z^{-N / 6}} S(z)
\end{array} .\right.
$$

By comparing the error transfer function gain in different strategies, the increased $G_{R E P}(z)$ in the denominator of the hybrid control system can reduce the $e_{i}$ gain significantly after adding repetitive control. Considering the resonant characteristics in the LCL filter, the gain of the PI controller cannot be extremely large; thus, the current tracking results of the PI controller are subject to certain restrictions. As long as the corrector of the repetitive controller is designed appropriately, the repetitive controller can improve the accuracy of current tracking without the influence of the PI controller gain.

\section{System Stability Analysis and Corrector Design}

To analyze the stability of the proposed fast-transient repetitive controller, the current closed loop transfer function of the hybrid controller is described as

$$
\left\{\begin{array}{c}
\frac{i_{\text {out }}(z)}{i_{\text {ref }}(z)}=\frac{\left\{G_{P I}(z)-z^{-N / 6}\left[Q G_{P I}(z)-S(z)\right]\right\} P(z)}{1-z^{-N / 6}[Q-S(z) P(z)]} \\
P(z)=\frac{G_{L C L}(z)}{1+G_{P I}(z) G_{L C L}(z)}
\end{array} .\right.
$$

$P(z)$ can be considered the equivalent controlled object of the repetitive controller. According to the small gain theorem, a sufficient condition [13, 25] for system stability can be provided as

$$
|H(z)|=|Q-S(z) P(z)|<1, z=e^{j \omega T_{s}}, \omega \in\left[0, \pi / T_{s}\right]
$$

where $T_{s}$ denotes the sampling period. Given that the equivalent controlled object contains resonant instability and phase delay, it must be corrected with an appropriately designed corrector $S(z)$. Furthermore, the corrector requires zero gain offset frequency response in a low-frequency band range; this condition does not affect the compensation current tracking performance.

In this study, a second-order low-pass filter and a zero phase-shift notch filter cascade connection $S(z)$ prevent the system stability condition from breaking at high frequencies [14]-[16]. The second-order low pass filter is required for high 
frequency attenuation, which greatly simplifies the controller design. A second-order filter with the transfer function (10) is selected to prevent the amplitude-frequency response within the current controller bandwidth from being affected. When crossover frequency $\omega_{n}=2 \pi \cdot 3750 \mathrm{rad} / \mathrm{s}$, damping coefficient $\xi=0.8$.

$$
\begin{aligned}
& F_{1}(s)=\frac{\omega_{n}^{2}}{s^{2}+2 \xi \omega_{n}+\omega_{n}^{2}} \\
& =\frac{(2 \pi \cdot 3750)^{2}}{s^{2}+2 \cdot 0.8 \cdot 2 \pi \cdot 3750+(2 \pi \cdot 3750)^{2}}
\end{aligned}
$$

Through Tustin transform $\left(f_{s}=15 \mathrm{kHz}\right)$, the discrete transfer function can be described as follows:

$$
F_{1}(z)=\frac{0.3625 z^{2}+0.725 z+0.3625}{z^{2}-0.2659 z+0.1842}
$$

Although the peak resonance of $G_{L C L}(s)$ shown in Fig. 3 is partly suppressed by passive damping in the branch of the filter capacitor, it does not satisfy the requirements for the stability of the overall system. Fig. 3 shows that the gain of resonant frequency still exceeds $0 \mathrm{~dB}$. Moreover, considering the addition gain of the current controller, system instability is easily caused. For this reason, an additional suppression method is required in the control algorithm. In this study, a zero phase-shift notch filter (denoted as $\left.F_{2}(z)\right)$ is adopted in the corrector to avoid the LCL filter resonance problem without degrading the steady-state performance of the repetitive controller. $F_{2}(z)$ is expressed as

$$
F_{2}(z)=\frac{z^{m}+a_{0}+z^{-m}}{2+a_{0}}
$$

where $a_{0}$ and $m$ are constants related to the shape and location of the notch filter, respectively [26]. Furthermore, the zero phase notch filter does not produce additional phase shift. The notch filter with a small value of $a_{0}$ causes a narrow stop band and allows a large amount of harmonic components to pass through the filter, which in turn results in better steady-state compensation performance of the repetitive controller. As shown in Fig. 3, the LCL filter resonant frequency is located around $5 \mathrm{kHz}$; thus, $F_{2}(z)$ can select parameters with $a_{0}=2, m=1$, which has a notch peak point near the corresponding LCL filter resonant frequency. The transfer function of $F_{2}(z)$ is expressed as

$$
F_{2}(z)=\frac{z+2+z^{-1}}{4} \text {. }
$$

Even with the corrector filter, the repetitive controller still

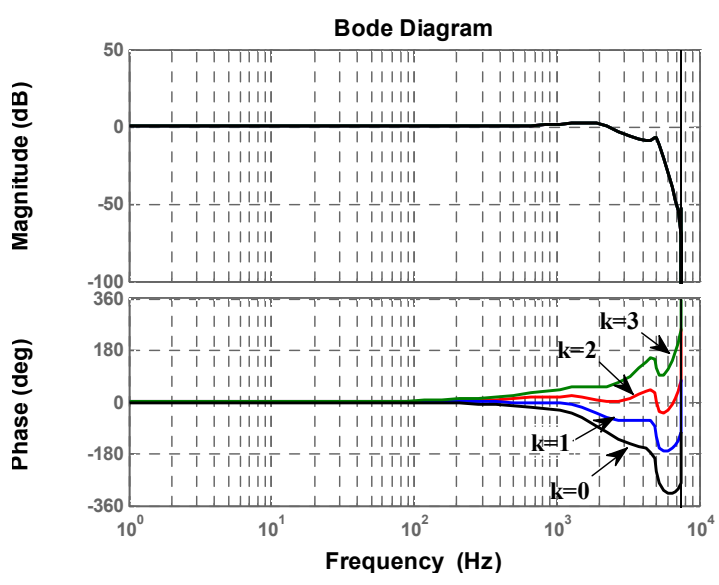

Fig. 10. Bode diagram of $S(z) P(z)$ with different values of $k$.

provides high gains in a low-frequency range and the magnitudes of the harmonic peaks in high-frequency regions are reduced to ensure system stability. However, the corrector filter can enhance system stability without causing much impact on the steady-state performance because the magnitude of the high-frequency harmonics is usually very small.

Phase lag must also be compensated for to maintain system stability and steady-state performance. Therefore, a leading element, $z^{k}$, is adopted to compensate for the delay caused by the calculation time in the digital control and to minimize the phase displacement of $S(z) P(z)$. The designed corrector of the fast-transient repetitive controller is

$$
S(z)=F_{1}(z) F_{2}(z) z^{k} .
$$

The frequency characteristics of $S(z) P(z)$ with different values of $k$ are shown in Fig. 10. Minimum phase displacement is attained with $k=2$. In a low-frequency band, the gain and phase lag of $S(z) P(z)$ are close to $0 \mathrm{~dB}$ and $0^{\circ}$, respectively, to ensure a small steady-state error. In addition, in consideration of the one beat delay in the digital control implementation, $k=3$ is selected.

The role of $Q$ in Equation (5) is to improve the system stability margin by reducing the peak gains of the repetitive controller in high-frequency ranges. In this study, $Q$ is selected as a close-to-unity constant 0.95 to guarantee a sufficient stability margin.

Using the aforementioned design results, the locus of $H(z)$ is plotted in Fig. 11.

In Fig. 11, $H(z)$ is always located inside the unity circle. Therefore, the proposed fast-transient repetitive controller with an appropriate designed corrector satisfies the stability condition.

\section{EXPERIMENTAL RESULTS AND ANALYSIS}




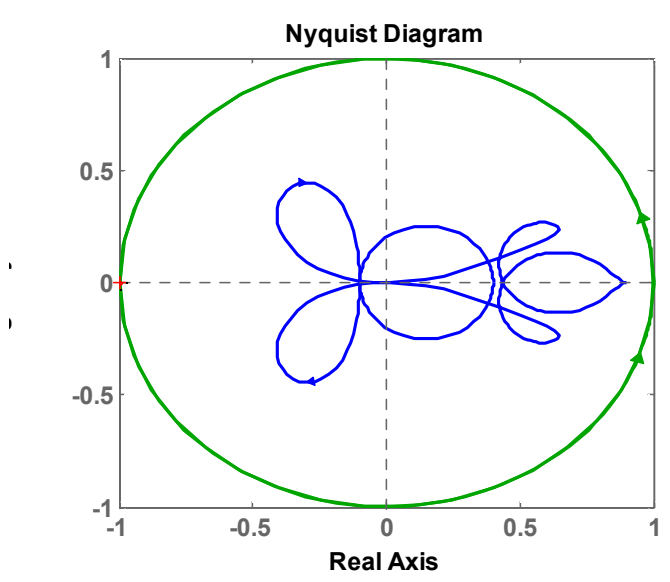

Fig. 11. Nyquist diagram of $H(z)$.

TABLE I

PARAMETERS OF THE Shunt APF Prototype

\begin{tabular}{|l|l|l|}
\hline \multicolumn{1}{|c|}{ Symbol } & \multicolumn{1}{c|}{ Parameter } & \multicolumn{1}{c|}{ Illustration } \\
\hline$V_{s}$ & $150 \mathrm{~V}$ & Utility voltage \\
\hline$U_{d c}$ & $300 \mathrm{~V}$ & DC side voltage of APF \\
\hline$f_{s w}$ & $15 \mathrm{kHz}$ & Switching frequency \\
\hline$f_{s}$ & $15 \mathrm{kHz}$ & Sampling frequency \\
\hline$L_{l}$ & $100 \mu \mathrm{H}$ & Inverter side inductor \\
\hline$L_{2}$ & $50 \mu \mathrm{H}$ & Grid side inductor \\
\hline$C_{f}$ & $30 \mu \mathrm{F}$ & Filter capacitor \\
\hline$R_{d}$ & $0.1 \Omega$ & Damping resistor \\
\hline$R_{\text {load }}$ & $5.8 \Omega$ & Diode rectifier resistive load \\
\hline$L_{\text {load }}$ & $1 \mathrm{mH}$ & Diode rectifier inductive load \\
\hline
\end{tabular}

To verify the aforementioned analysis, experiments are implemented on a three-phase LCL filter-based shunt APF prototype, which is depicted in Fig. 1. The key parameters of the prototype are listed in Table 1. The control platform is implemented on a TMS320F28335 DSP control board. The insulated gate bipolar transistor (IGBT) module FF300R12MS4 from Infineon is used in the main circuit power inverter.

\section{A. Steady-state Performance of PCC Current}

Fig. 12(a) shows the waveform of the grid current with nonlinear RL load before the shunt APF is operated. The frequency spectrum of the grid current is shown in Fig. 12(b). The total harmonic distortion (THD) of the grid current is approximately $26.1 \%$.

Fig. 13 shows the experimental results of the steady-state grid current with a traditional PI current controller. As shown in Figs. 12 and 13, reducing the THD of the grid current from $26.1 \%$ to $8.5 \%$ is possible by means of a traditional PI controller. The components of the 5th, 7th, 11th, and 13th harmonic currents are greatly suppressed, and the shunt APF with a traditional PI controller allows some other harmonic currents into the grid current. The traditional PI controller is

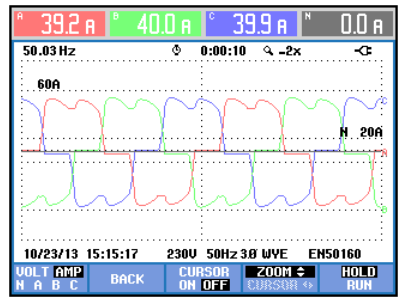

(a) Phase currents.

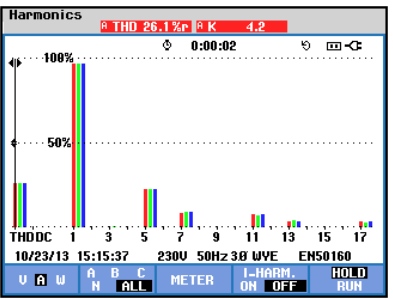

(b) Magnitude spectrum.
Fig. 12. Waveform of the grid current without shunt APF.

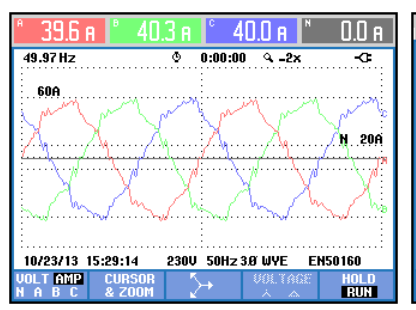

(a) Phase currents.

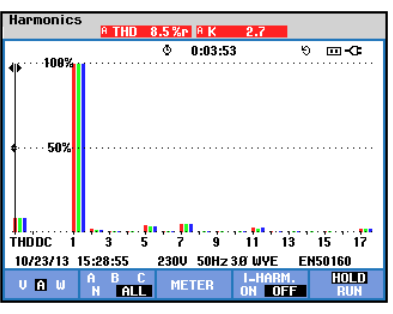

(b) Magnitude spectrum.
Fig. 13. Steady-state performance with a traditional PI controller.

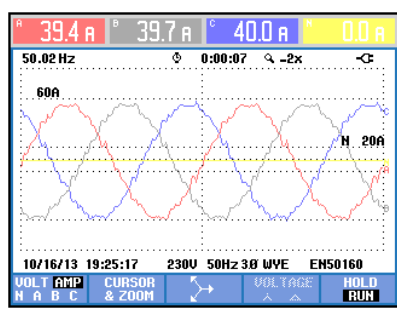

(a) Phase currents.

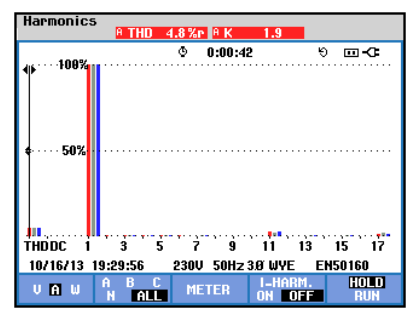

(b) Magnitude spectrum.
Fig. 14. Steady-state performance with a PR controller.

unable to effectively compensate for the harmonic currents because of its control bandwidth limitation.

The experimental results of the PR controller [12] whose regulators tuned on resonance frequencies 6th, 12th, and 18th are shown in Fig. 14. When the PR controller is employed, the grid current exhibits better steady-state performance and THD is reduced to $4.8 \%$. However, the PR controller does not generate high gains in harmonics above the 18th. The high-order harmonic current compensation performance is thus ineffective.

An experimental test with a conventional repetitive control algorithm under the same condition is shown in Fig. 15. The harmonic currents are effectively compensated, and the grid current is almost sinusoidal with a low THD of approximately $3.7 \%$. This result proves that the repetitive controller has a better steady filtering capability compared with the traditional PI controller.

Fig. 16(a) shows the waveforms of the grid current when a hybrid current controller is employed. The PI and fast-transient repetitive controllers are connected in parallel when the shunt APF operates. As shown in Fig. 16(b), the THD of the grid current is $3.6 \%$ when the APF filter with a fast-transient repetitive controller is used. This result confirms that the 


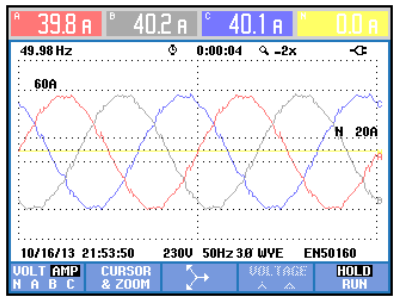

(a) Phase currents.

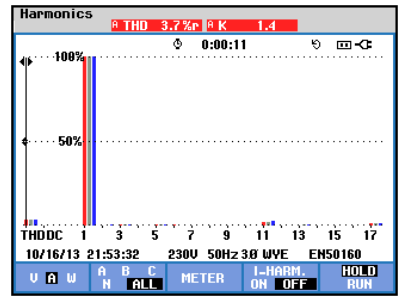

(b) Magnitude spectrum.
Fig. 15. Steady-state performance with a conventional repetitive controller.

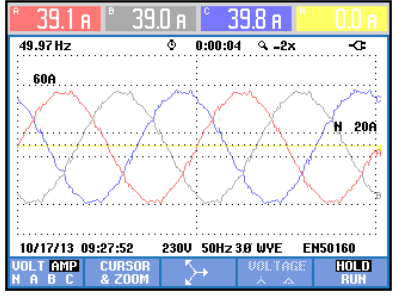

(a) Phase currents.

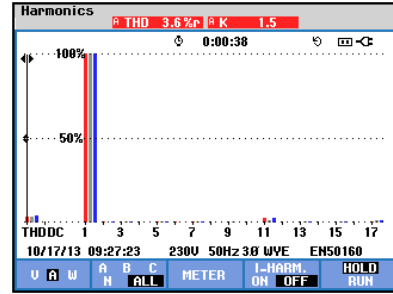

(b) Magnitude spectrum.
Fig. 16. Steady-state performance with the proposed fast-transient repetitive controller.

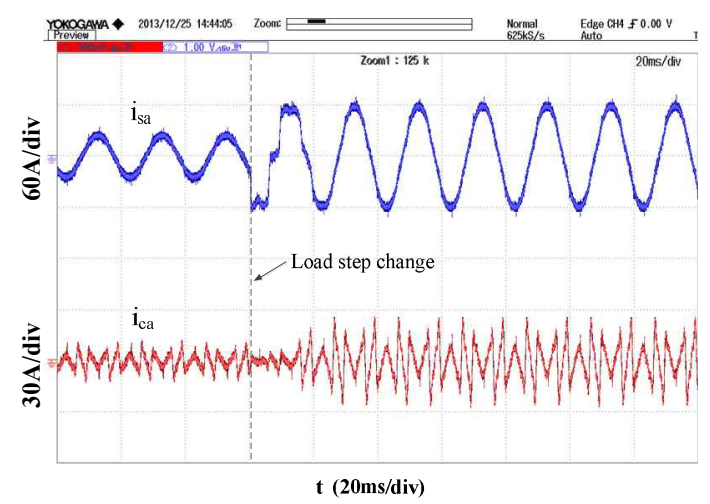

Fig. 17. Transient responses when a conventional repetitive controller is used.

performance of the proposed controllers is similar to that of the conventional one in steady state conditions.

\section{B. Dynamic Responses under Load Step Change}

Fig. 17 illustrates the experimental waveforms of the dynamic response when a conventional repetitive controller is employed and when the load varies from light to heavy. The compensation current tracks the load harmonic current within nearly two fundamental cycles.

Fig. 18 shows the dynamic response of the fast-transient repetitive controller. The filter current quickly responds to compensate for the harmonic currents in the load and to ensure that the supply current is sinusoidal. As shown in Figs. 17 and 18 , the current tracking error recovers to its steady-state value in approximately $10 \mathrm{~ms}$, which is much shorter than that in the conventional controller. As a result, the fast transient responses of the proposed controller are verified.

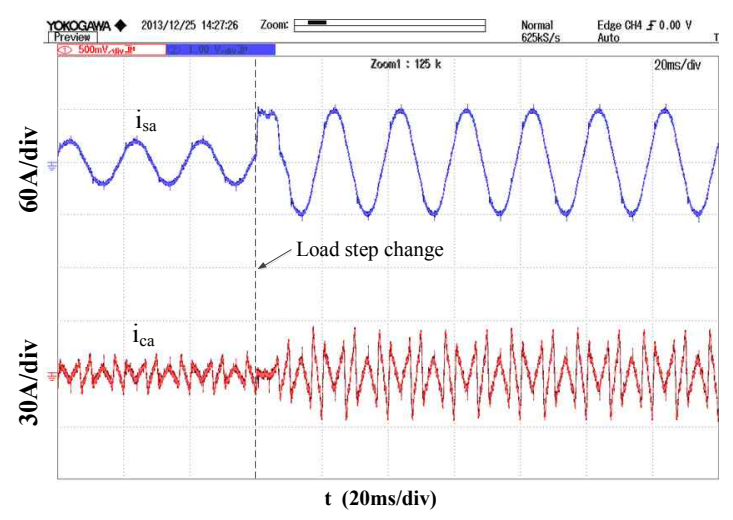

Fig. 18. Transient responses when a fast-transient repetitive controller is used.

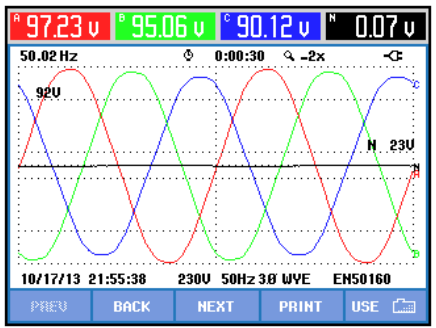

Fig. 19. Waveform of unbalanced three-phase grid voltages.

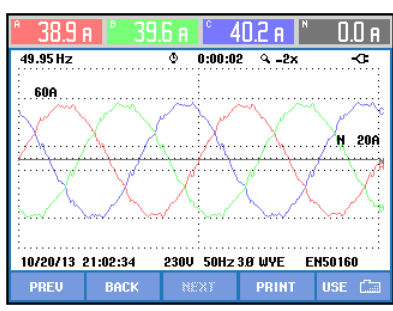

(a) Phase currents.

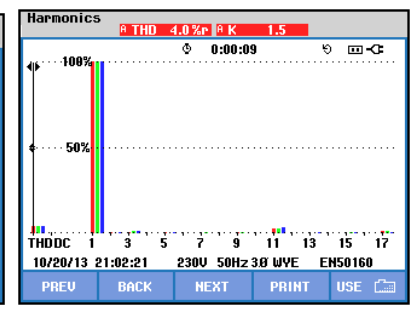

(b) Magnitude spectrum
Fig. 20. Steady-state performance with the fast-transient repetitive controller under an unbalanced grid condition.

\section{Performance of PCC Current under Unbalanced Supply Voltage}

The majority of previous studies assumed that the supply voltage is ideally balanced; however, this voltage condition is rare in practical grid conditions. To verify the effectiveness of the proposed control algorithm under unbalanced conditions, experiments are conducted where the supply voltages are injected with negative components that are $10 \%$ of the magnitude of the fundamental component.

The waveform of the unbalanced three-phase grid voltage is shown in Fig. 19.

As shown in Fig. 20, the harmonic compensation performance of APF does not diminish under the unbalanced grid voltage condition. The grid current is nearly sinusoidal, which is similar to the results shown in Fig. 20 where an ideal balanced voltage is provided. Only small increases were observed in the THD factors, that is, approximately $4.0 \%$ for the same case of nonlinear RL load. 


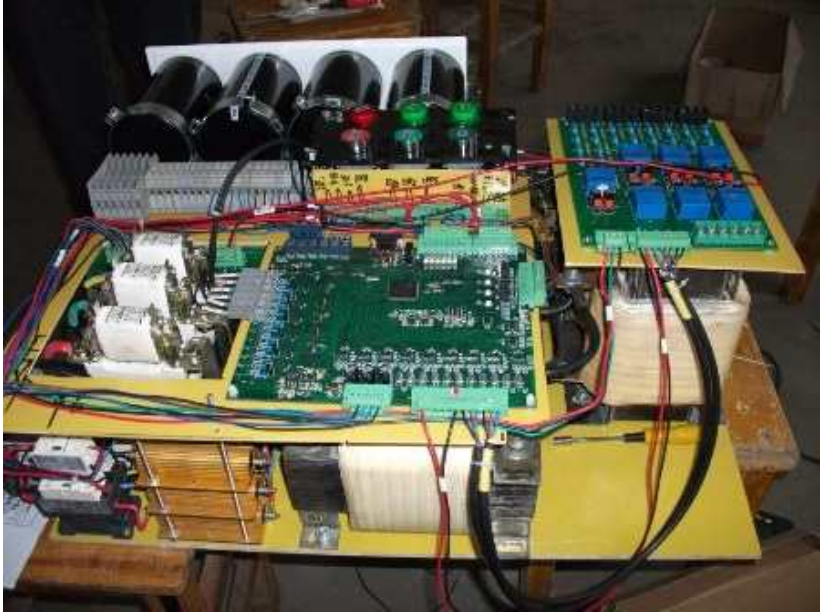

Fig. 21. Implemented LCL filter-based shunt APF system.

As a result, the proposed fast-transient repetitive controller obtains a remarkably fast transient response and a satisfactory steady filtering capability.

\section{CONCLUSIONS}

A fast-transient repetitive controller for a three-phase LCL filter-based shunt APF was proposed in this study. This controller minimizes the repetitive control time delay to one-sixth of the fundamental period and improves the dynamic responses without sacrificing high steady-state performance. In the hybrid control scheme, the repetitive controller improves the tracking accuracy and eliminates the steady state error. The PI controller guarantees the dynamic response and current tracking speed of APF. Furthermore, the strategy suppresses the resonance problem of the LCL filter by using an appropriately designed corrector. The experimental results verify the feasibility and validity of the proposed strategy. Owing to its fast transient response and high tracking accuracy, this strategy is also appropriate for three-phase grid-connected inverters, such as static synchronous compensators or uninterruptible power supplies.

\section{ACKNOWLEDGMENT}

This work was supported in part by the National Natural Science Foundation of China (Project no. 51177150) and the National Key Basic Research Program of China (Project no. 973) under Grant no. 2013CB035604.

\section{REFERENCES}

[1] H. Akagi, "Active harmonic filters," in Proc. the IEEE, Vol. 93, No. 12, pp. 2128-2141, Dec. 2005.

[2] M. Liserre, F. Blaabjerg, and S. Hansen, "Design and control of an LCL-filter-based three-phase active rectifier," IEEE Trans. Ind. Appl., Vol. 41, No.5, pp. 1281-1291, Sep./Oct. 2005.

[3] T. Yi, C. L. Poh, W. Peng, H. C. Fook, G. Feng, and F.
Blaabjerg, "Generalized design of high performance shunt active power filter with output LCL filter," IEEE Trans. Ind. Electron., Vol. 59, No. 3, pp. 1443-1452, Mar. 2012.

[4] D. Wojciechowski and R. Strzelecki, "High power, zero ripples active filtering system with power modules operating in parallel," in Electronics Conference (BEC), pp. 305-308, 2010.

[5] O. Vodyakho and C. C. Mi, "Three-level inverter-based shunt active power filter in three-Phase three-wire and four-wire systems," IEEE Trans. Power Electron., Vol. 24, No. 5, pp. 1350-1363, May 2009.

[6] M. I. M. Montero, E. R. Cadaval, and F. B. Gonzalez, "Comparison of control strategies for shunt active power filters in three-phase four-wire systems," IEEE Trans. Power Electron., Vol. 22, No. 1, pp. 229-236, Jan. 2007.

[7] A. Luo, Z. Shuai, W. Zhu, R. Fan, and C. Tu, "Development of hybrid active power filter based on the adaptive fuzzy dividing frequency-control method," IEEE Trans. Power Del., Vol. 24, No. 1, pp. 424-432, Jan. 2009.

[8] J. Matas, L. G. de Vicuna, J. Miret, J. M. Guerrero, and M. Castilla, "Feedback linearization of a single-phase active power filter via sliding mode control," IEEE Trans. Power Electron., Vol. 23, No. 1, pp. 116-125, Jan. 2008.

[9] B. Singh and J. Solanki, "An implementation of an adaptive control algorithm for a three-phase shunt active filter, “ IEEE Trans. Ind. Electron., Vol. 56, No.8, pp. 2811-2820, Aug. 2009.

[10] I. J. Gabe, V. F. Montagner, and H. Pinheiro, "Design and implementation of a robust current controller for VSI connected to the grid through an LCL filter," IEEE Trans. Power Electron., Vol.24, No. 6, pp. 1444-1452, Jun. 2009.

[11] Z. Wang, C. Xie, C. He, and G. Chen, "A waveform control technique for high power shunt active power filter based on repetitive control algorithm," in Proc. IEEE APEC, pp. 361-366, 2010.

[12] R. I. Bojoi, G. Griva, V. Bostan, M. Guerriero, F. Farina, and F. Profumo, "Current control strategy for power conditioners using sinusoidal signal integrators in synchronous reference frame," IEEE Trans. Power Electron., Vol. 20, No.6, pp. 1402-1412, Nov. 2005.

[13] B. Zhang, D. Wang, K. Zhou, and Y. Wang, "Linear phase lead compensation repetitive control of a CVCF PWM inverter," IEEE Trans. Ind. Electron., Vol. 55, No. 4, pp. 1595-1602, Apr. 2008.

[14] G. Escobar, A. A. Valdez, J. Leyva-Ramos, and P. Mattavelli, "Repetitive-based controller for a UPS inverter to compensate unbalance and harmonic distortion," IEEE Trans. Ind. Electron., Vol. 54, No. 1, pp. 504-510, Feb. 2007.

[15] T. Xin, K. M. Tsang, and W. L. Chan, "A power quality compensator with DG interface capability using repetitive control," IEEE Trans. Energy Convers., Vol. 27, No. 2, pp. 213-219, Jun. 2012.

[16] A. Garcia-Cerrada, O. Pinzon-Ardila, V. Feliu-Batlle, P. Roncero-Sanchez, and P. Garcia-Gonzalez, "Application of a repetitive controller for a three-phase active power filter," IEEE Trans. Power Electron., Vol. 22, No.1, pp. 237-246, Jan. 2007.

[17] R. Costa Castello, R. Grino, R. Cardoner Parpal, and E. Fossas, "High-performance control of a single-phase shunt active filter," IEEE Trans. Control Syst. Technol., Vol. 17, No. 6, pp. 1318-1329, Nov. 2009.

[18] J. Miret, M. Castilla, J. Matas, J. M. Guerrero, and J. C. Vasquez, "Selective harmonic-compensation control for single-phase active power filter with high harmonic 
rejection," IEEE Trans. Power Electron., Vol. 56, No.8, pp. 3117-3127, Aug. 2009.

[19] Z. Zou, Z. Wang, M. Cheng, and Y. Yang, "Active power filter for harmonic compensation using a digital dual-mode-structure repetitive control approach," in Proc. IEEE PEDG, pp. 161-166, 2012.

[20] Z. Zeng, J. Yang, and N. Yu, "Research on PI and repetitive control strategy for shunt active power filter with LCL filter," in Proc. IEEE IPEMC, pp. 2833-2837, 2012.

[21] R. Costa-Castello and R. Grino and E. Fossas, "Odd-harmonic digital repetitive control of a single-phase current active filter," IEEE Trans. Power Electron., Vol. 19, No. 4, pp. 1060- 1068, Jul. 2004.

[22] R. Grino, R. Cardoner, R. Costa-Castello, and E. Fossas, "Digital repetitive control of a three-phase four-wire shunt active filter," IEEE Trans. Ind. Electron., Vol. 54, No.3, pp. 1495-1503, Jun. 2007.

[23] W. Lu, K. Zhou, M. Cheng, Y. Yang, and S. Fan, "Parallel structure repetitive controller for CVCF three-phase PWM inverter," in Proc. IEEE APEC, pp. 207-210, 2012.

[24] G. Escobar, P. G. Hernandez-Briones, P. R. Martinez, M. Hernandez-Gomez, and R. E. Torres-Olguin, "A repetitive-based controller for the compensation of $61 \pm 1$ harmonic components," IEEE Trans. Ind. Electron., Vol. 55, No.8, pp. 3150-3158, Aug. 2008.

[25] S. Jiang, D. Cao, Y. Li, J. Liu, and F. Z. Peng, "Low-THD, fast-transient, and cost-effective synchronous-frame repetitive controller for three-phase UPS inverters," IEEE Trans. Power Electron., Vol. 27, No.6, pp. 2994-3005, Jun. 2012.

[26] G. F. Teng, G. C. Xiao, L. L. Hu, Y. Lu, and Y. R. Kafle, "Control Strategy Based on Equivalent Fundamental and Odd Harmonic Resonators for Single-Phase DVRs," Journal of Power Electronics, Vol. 12, No.4, pp. 654-663, Jul. 2012.

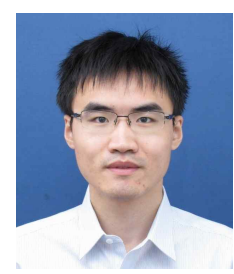

Zheng Zeng was born in Hunan, China, in 1987. He received his B.S. degree from Central South University, Changsha, China, in 2009. He is pursuing his Ph.D. degree in electrical engineering at Zhejiang University, Hangzhou, China. His research interests include power quality and control technology, power electronics and electric drives, and renewable energy generation.

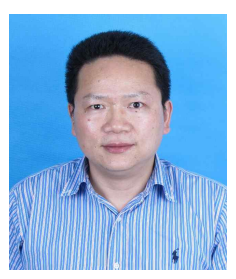

Jia-Qiang Yang was born in Jiangsu, China, in 1970 . He received his Ph.D. degree in electrical engineering from Zhejiang University, Hangzhou, China, in 2004. He has been an associate professor at the College of Electrical Engineering, Zhejiang University, Hangzhou, China, since 2006. He was a research fellow at the National University of Singapore, Singapore, from March 2011 to April 2012. His research interests include power electronics, electric machine drives, and control strategies.

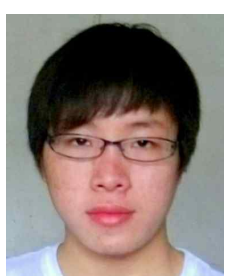

Shi-Lan Chen was born in Jiangxi, China, in 1990. He received his B.S. degree in electrical engineering from North University of China, Taiyuan, China, in 2011. He is pursuing his M.S. degree in electrical engineering at Zhejiang University, Hangzhou, China. His current research interests include harmonic and reactive power compensation in power grids.

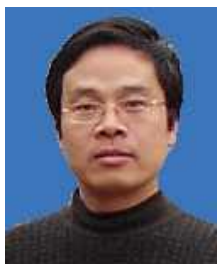

Jin Huang received his B.Sc. degree from Zhejiang University, Hangzhou, China, in 1982 and his Ph.D. degree in electrical engineering from the National Polytechnic Institute of Toulouse, Toulouse, France, in 1987. He worked as an associate professor at the College of Electrical Engineering, Zhejiang University, Hangzhou, China, from 1987 to 1994 . He has been a professor in Zhejiang University since 1994. He is engaged in research on electrical machines, AC drives, multiphase machines, and control strategies. 